\title{
绝缘性能检测在 CSSD 中的使用方法研究
}

\section{Research on the Application Method of Insulation Performance Test in CSSD 周文华 黄轶斐 \\ Wenhua Zhou Yifei Huang}

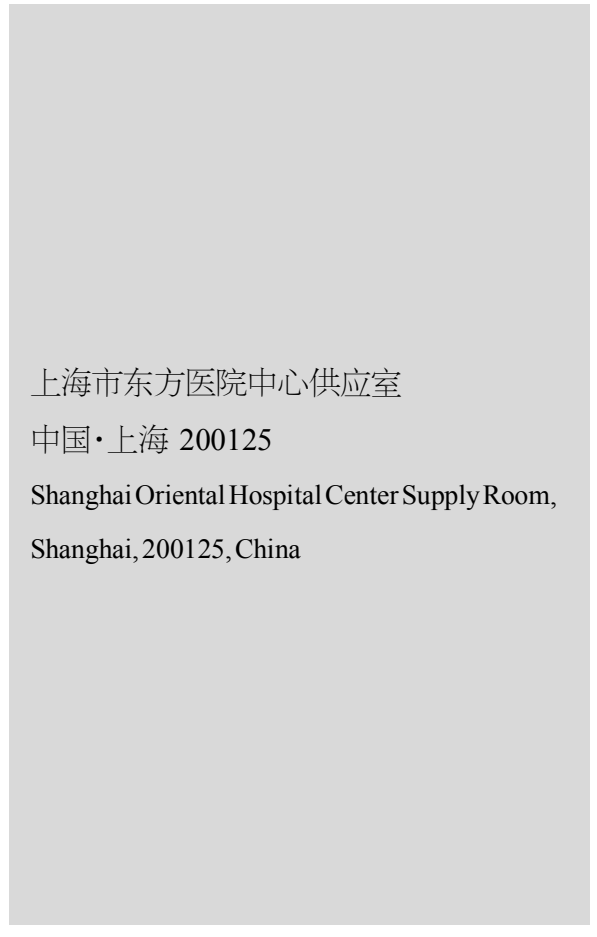

\section{1 引言}

电外科术是以高频电流的凝固和烧灼作用施行的手术治 疗方法,一般对皮肤的作用是将热传导给组织,或通过电流在 组织产生热反应。在皮肤外科手术中, 常用的电外科方法是电 凝和电切割, 与普通外科的使用方法相同, 而电灼、电干燥和 电烙在皮肤科可与其他治疗方法结合使用。由于手术器械必 须经过消毒供应中心 (central sterile supply department,CSSD ) 进行清洗、消毒、灭菌, 各种机械、物理和化学接触造成的损 耗,会使绝缘层发生破损。电外科手术设备是手术室不可缺少 的设备, 电外科手术器械已经呈现出专用化制造的发展趋势, 并广泛应用于皮肤科、妇科、心脏外科、整形科、眼科、脊柱科、 耳鼻喉科、领面外科、骨科、泌尿科、神经外科、一般外科手术 以及某些牙科手术 ${ }^{[1]}$ 。在供应室内进行全面检测, 避免了手术 器械漏电的风险, 防止不良事件的发生, 保证了患者的安全。

\section{2 电外科手术与手术器械}

\section{1 电外科手术原理}

电外科手术使用的高频电刀是一个变频变压器, 将 $220 \mathrm{~V} /$
$50 \mathrm{~Hz}$ 的低压低频电流经变频变压, 功率放大转换为频率 400 $1000 \mathrm{kHz}$ 、电压为几千甚至上万伏的高频电流, 可以在人体组 织上产生切割和凝血的作用。切割时电流使细胞膨胀、破裂、 汽化, 电凝时使细胞减少出血。

\section{2 电外科手术器械}

电外科手术器械使用的技术分为热技术和冷技术。热技 术指的是利用电流及其换能后的形式所产生的热作用而工作 的技术, 此类器械包括基础电刀、双极电刀、氩气刀、大血管闭 合系统、超声刀、超声乳化吸引刀、射频刀等; 冷技术指的是利 用水流或制冷气体所产生的冷作用而工作的技术，此类器械 包括水刀、冷冻器械等。

\section{3 电外科手术器械绝缘保护层}

为防止患者被高压高频电流灼伤，常用的绝缘层材料有 低密度聚乙烯、离子聚合物、高密度聚乙烯和聚氯乙烯等。一 般电外科手术器械金属部分的外层都包裹着绝缘层，有效隔 阻使用时通过器械传导的高频高压电流, 以免患者和医生意 外灼伤。一旦电外科手术器械的绝缘层发生破损, 便会威胁到 医生和患者的安全 ${ }^{[2]}$ 


\section{3 电外科手术风险与手术器械漏电}

\section{1 电外科手术风险}

电外科的主要风险包括手术相关风险、对手术室环境造 成的潜在风险、漏电及电相关的风险等。

3.1 .1 与手术相关的风险

凝血不止是因为电凝产生的焦痂不牢固，一旦焦痂脱落 都会再出血。氩气等离子电凝是更安全、可靠的电凝器械。

3.1 .2 对周围组织的热损伤

使用氩气等离子电凝或超声刀代替单极电刀分离组织, 能有效减少组织的热损伤。

3.1 .3 伤口愈合不良与脂肪液化

对于肥胖者而言, 皮下脂肪较多或富含脂肪的组织, 电刀 使用过程中会造成脂肪液化，影响伤口愈合和导致无菌性炎 症的发生。

\subsection{4 与手术室环境相关的风险}

手术室是一个富氧环境, 存在大量的易燃物品, 这是电刀 致手术室火花的重要因素。

3.1 .5 漏电及电相关的风险

电刀负极板放置位置不妥, 会引起电灼伤。直接耦合、电 容耦合及绝缘层失败, 这三者是导致患者术中意外损伤的重 要原因。

\section{2 电外科手术漏电风险}

\subsection{1 绝缘层损坏}

手术器械绝缘层破损, 主要发生在器械及器械连接线上。 器械表面绝缘层发生损坏, 可能导致患者体内意外灼伤; 器械 连接线发生破损,可能使患者肢体或医生发生意外。根据电外 科的工作原理, 绝缘层破损面积越小, 泄漏电流密度越大, 从 而造成的损伤也越严重 ${ }^{[3]}$ 。为避免器械损坏, 通道用高压气枪 吹干, 在干燥柜 (温度设置为 $65 \sim 75^{\circ} \mathrm{C}$ ) 内干燥, 时间 $\leqslant 30 \mathrm{~min} 。$ 马达、手柄及钻头禁止超声清洗与润滑干燥, 用绝缘检测仪对 马达及连接线进行绝缘性能检测 ${ }^{[4]}$ 。供应室专科技术人员、外 科医生和护士需要对手术器械的完整性负责。如果一种仪器 被怀疑有缺陷, 必须从循环系统中移除, 直到修理或更换后才 可使用 ${ }^{[5]}$ 。

\subsection{2 电容耦合与直接耦合}

当电流通过器械电极以及绝缘体传递到相邻导体时, 会 发生电容耦合。当器械电极放置得太靠近或接触金属器械或
其他器械时,腹腔镜手术期间会发生直接耦合。

3.2.3 中性电极灼伤

电极包括硅胶、单片式、双片式。在单极技术运用的过程 中, 手柄回收释放出的电流形成完整的电流回路,如果发生粘 贴不良或接触面积过小均会导致电流过大、电阻增加而发生 灼伤。

\section{3 电外科器械漏电导致的不良事件}

电外科手术器械绝缘层破损可能导致病人体内意外灼 伤。电绝缘失败造成的伤害包括腹腔内出血、胃肠道穿孔、血 管破裂、不明原因的发热、白血球增多及其他不明原因症状 ${ }^{[6]}$ 。

\section{4 使用绝缘检测仪进行器械绝缘性能结果检}

\section{测和保护功能措施}

本院使用欧洲医疗生产的 HiPOT150 型绝缘检测仪对院 内单极器械及双极器械进行绝缘性能检测, 并将其中发现存 在绝缘层性能问题的器械进行替换。被测设备保护地与电源 地线之间的电阻被称为接地阻抗，是针对设备漏电时确保人 碰触外壳不会触电而要求的电气指标, 保护了检测者不被漏 电所伤害, 保证了医务人员的安全 ${ }^{[7]}$ 。

\section{4 结语}

本文研究结果显示, 在保障手术器械安全供应的同时,应 该确保手术器械功能完好性的检测, 确保手术器械的安全使 用。使用电外科手术器械绝缘检测仪对电外科手术器械的绝 缘层进行功能检测, 是杜绝手术器械不良事件发生的良好解 决方案, 能够提高手术质量,保障患者的安全。

\section{参考文献}

[1]陈孝平,汪建平.外科学[M].北京: 人民卫生出版社,2013.

[2]娄正林,张坚,张婧.腹腔镜手术中器械对人体的损伤及预防 [J]. 生物医学工程学进展,2017,38(1):54-56.

[3]刘东岩,郭大为,王爽,等. 有源腔镜器械的绝缘性能检测及破损 原因分析[J].中国医学装备,2018,15(11):54-57.

[4]乔秀芹, 徐瑞芸, 景阳阳.耳.鼻咽喉手术动力系统清洗保养方法 探讨[J].中国消毒学杂志,2017,34(4):370-371.

[5]Spruce L,Braswell ML.Implementing AORN Recommended Practices for Electrosurgery[J].AORN J,2012,95(3):379-384.

[6]袁园,刘作辉.电外科手术器械绝缘性能检测[J].中国医学装备, 2018,15(5):123-127.

[7]崔志刚,刘坚,高虹. 医疗设备电气安全的检测与探讨 [J]. 医疗卫 生装备,2010,31(5):110-111+113. 